\title{
Pydra - a flexible and lightweight dataflow engine for scientific analyses
}

\author{
Dorota Jarecka $^{\ddagger *}$, Mathias Goncalves ${ }^{\mathbb{I} \ddagger}$, Christopher J. Markiewicz $Z^{\mathbb{I}}$, Oscar Esteban ${ }^{\mathbb{I I}}$, Nicole Lo ${ }^{\ddagger}$, Jakub \\ Kaczmarzyk $^{\S \ddagger}$, Satrajit Ghosh
}

\begin{abstract}
This paper presents a new lightweight dataflow engine written in Python: Pydra. Pydra is developed as an open-source project in the neuroimaging community, but it is designed as a general-purpose dataflow engine to support any scientific domain. The paper describes the architecture of the software, as well as several useful features, that make Pydra a customizable and powerful dataflow engine. Two examples are presented to demonstrate the syntax and properties of the package.
\end{abstract}

Index Terms—dataflow engine, scientific workflows, reproducibility

\section{Introduction}

Scientific workflows often require sophisticated analyses that encompass a large collection of algorithms. The algorithms, that were originally not necessarily designed to work together, and were written by different authors. Some may be written in Python, while others might require calling external programs. It is a common practice to create semi-manual workflows that require the scientists to handle the files and interact with partial results from algorithms and external tools. This approach is conceptually simple and easy to implement, but the resulting workflow is often time consuming, error-prone and difficult to share with others. Consistency, reproducibility and scalability demand scientific workflows to be organized into fully automated pipelines. This was the motivation behind Pydra - a new dataflow engine written in Python, that is presented in this paper.

The Pydra package is a part of the second generation of the Nipype ecosystem $\left(\left[\mathrm{GBM}^{+} 11\right]\right.$, [Dev]) --- an open-source framework that provides a uniform interface to existing neuroimaging software and facilitates interaction between different software components. The Nipype project was born in the neuroimaging community, and has been helping scientists build workflows for a decade, providing a uniform interface to such neuroimaging packages as FSL [WJP+09], ANTs [ATS09], AFNI [Cox96], FreeSurfer [DFS99] and SPM [FAK $\left.{ }^{+} 07\right]$. This flexibility has made it an ideal basis for popular preprocessing tools, such as fMRIPrep [OEG19] and C-PAC [C-P]. The second generation of Nipype ecosystem is meant to provide additional flexibility and is

* Corresponding author: djarecka@gmail.com

¥ Massachusetts Institute of Technology, Cambridge, MA, USA

II Stanford University, Stanford, CA, USA

§ Stony Brook University School of Medicine, Stony Brook, NY, USA

Copyright $@ 2020$ Dorota Jarecka et al. This is an open-access article distributed under the terms of the Creative Commons Attribution License, which permits unrestricted use, distribution, and reproduction in any medium, provided the original author and source are credited. being developed with reproducibility, ease of use, and scalability in mind. Pydra itself is a standalone project and is designed as a general-purpose dataflow engine to support any scientific domain.

The goal of Pydra is to provide a lightweight dataflow engine for computational graph construction, manipulation, and distributed execution, as well as ensuring reproducibility of scientific pipelines. In Pydra, a dataflow is represented as a directed acyclic graph, where each node represents a Python function, execution of an external tool, or another reusable dataflow. The combination of several key features makes Pydra a customizable and powerful dataflow engine:

- Composable dataflows: Any node of a dataflow graph can be another dataflow, allowing for nested dataflows of arbitrary depths and encouraging creating reusable dataflows.

- Flexible semantics for creating nested loops over input sets: Any Task or dataflow can be run over input parameter sets and the outputs can be recombined (similar concept to Map-Reduce model [DG04], but Pydra extends this to graphs with nested dataflows).

- A content-addressable global cache: Hash values are computed for each graph and each Task. This supports reusing of previously computed and stored dataflows and Tasks.

- Support for Python functions and external (shell) commands: Pydra can decorate and use existing functions in Python libraries alongside external command line tools, allowing easy integration of existing code and software.

- Native container execution support: Any dataflow or Task can be executed in an associated container (via Docker or Singularity) enabling greater consistency for reproducibility.

- Auditing and provenance tracking: Pydra provides a simple JSON-LD -based message passing mechanism to capture the dataflow execution activties as a provenance graph. These messages track inputs and outputs of each task in a dataflow, and the resources consumed by the task.

Pydra is a pure Python 3.7+ package with a limited set of dependencies, which are themselves only dependent on the Python Standard library. It leverages type annotation and AsyncIO in its core operations. Pydra uses the attr package for extended annotation and validation of inputs and outputs of tasks, the cloudpickle package to pickle interactive task definitions, and the pytest testing framework. Pydra is intended to help scientific workflows which rely on significant file-based operations and 
which evaluate outcomes of complex dataflows over a hyperspace of parameters. It is important to note, that Pydra is not a framework for writing efficient scientific algorithms or for use in applications where caching and distributed execution are not necessary. Since Pydra relies on a filesystem cache at present, it is also not designed for dataflows that need to operate purely in memory.

The next section will describe the Pydra architecture --- main package classes and interactions between them. The Key Features section focuses on a set of features whose combination distinguishes Pydra from other dataflow engines. The paper concludes with a set of applied examples demonstrating the power and utility of Pydra, and short discussion on the future directions.

\section{Architecture}

Pydra architecture has three core components: Task, Submitter and Worker. Tasks form the basic building blocks of the dataflow, while Submitter orchestrates the dataflow execution model. Different types of Workers allow Pydra to execute the task on different compute architectures. Fig. 1 shows the Class hierarchy and interaction between them in the present Pydra architecture. It was designed this way to decouple Tasks and Workers. In order to describe Pydra's most notable features in the next section, we briefly describe the role of each of these classes.

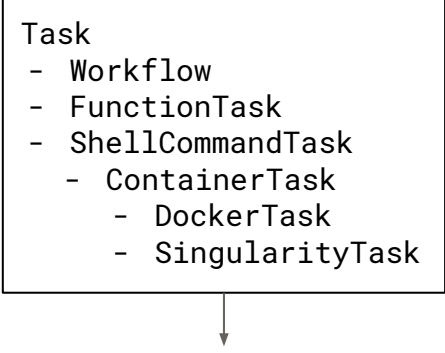

Submitter

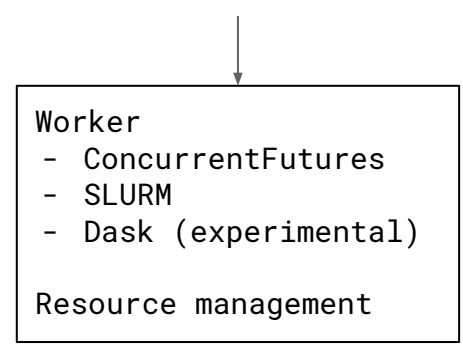

Fig. 1: A schematic presentation of principal classes in Pydra.

\section{Dataflows Components: Task and Workflow}

A Task is the basic runnable component of Pydra and is described by the class TaskBase. A Task has named inputs and outputs, thus allowing construction of dataflows. It can be hashed and executes in a specific working directory. Any Pydra's Task can be used as a function in a script, thus allowing dual use in Pydra's Workflows and in standalone scripts. There are several classes that inherit from TaskBase and each has a different application:

- FunctionTask is a Task that executes Python functions. Most Python functions declared in an existing library, package, or interactively in a terminal can be converted to a FunctionTask by using Pydra's decorator mark.task.

import numpy as np

from pydra import mark

fft $=$ mark. annotate $(\{' a '$ : np.ndarray,

fft_task = mark.task (fft) () 'return': float ) (np.fft.fft)

result $=f f t \_t a s k(a=n p \cdot r a n d o m \cdot r a n d(512))$

fft_task is now a PydraTask and result will contain a Pydra's Result object. In addition, the user can use Python's function annotation or another Pydra decoratormark. annotate in order to specify the output. In the following example, we decorate an arbitrary Python function to create named outputs:

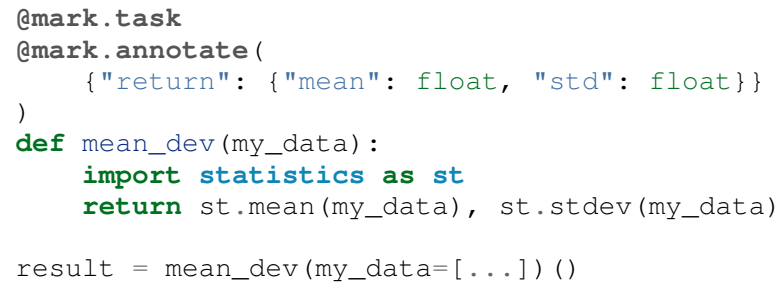

When the Task is executed result.output will contain two attributes: mean and std. Named attributes facilitate passing different outputs to different downstream nodes in a dataflow.

- ShellCommandTask is a Task used to run shell commands and executables. It can be used with a simple command without any arguments, or with specific set of arguments and flags, e.g.:

ShellCommandTask (executable="pwd")

ShellCommandTask (executable="ls", args="my_dir")

The Task can accommodate more complex shell commands by allowing the user to customize inputs and outputs of the commands. One can generate an input specification to specify names of inputs, positions in the command, types of the inputs, and other metadata. As a specific example, FSL's BET command (Brain Extraction Tool) can be called on the command line as:

bet input_file output_file -m

Each of the command argument can be treated as a named input to the ShellCommandTask, and can be included in the input specification. As shown next, even an output is specified by constructing the out_file field form a template:

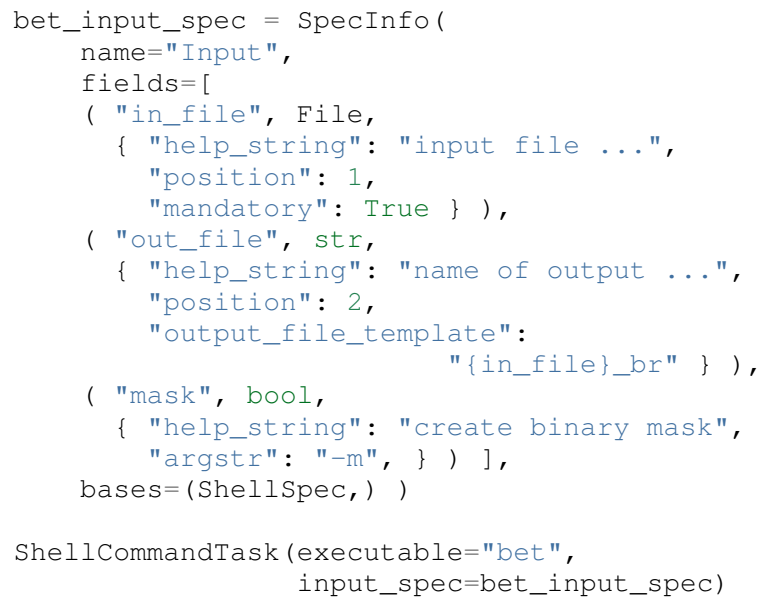


Outputs can also be specified separately using a similar output specification.

- ContainerTask class is a child class of ShellCommandTask and serves as a parent class for DockerTask and SingularityTask. Both Container Tasks run shell commands or executables within containers with specific user defined environments using Docker [doc] and Singularity [sin] software respectively. This might be extremely useful for users and projects that require environment encapsulation and sharing. Using container technologies helps improve scientific workflows reproducibility, one of the key concept behind Pydra.

These Container Tasks can be defined by using DockerTask and SingularityTask classes directly, or can be created automatically from ShellCommandTask, when an optional argument container_info is used when creating a Shell Task. The following two types of syntax are equivalent:

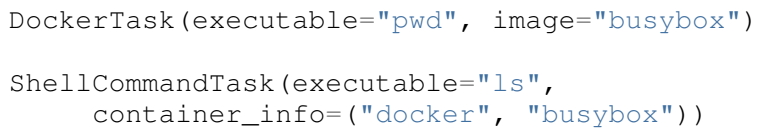

- Workflow - is a subclass of Task that provides support for creating Pydra dataflows. As a subclass, a Workflow acts like a Task and has inputs, outputs, is hashable, and is treated as a single unit. Unlike Tasks, workflows embed a directed acyclic graph. Each node of the graph contains a Task of any type, including another Workflow, and can be added to the Workflow simply by calling the add method. The connections between Tasks are defined by using so called Lazy Inputs or Lazy Outputs. These are special attributes that allow assignment of values when a Workflow is executed rather than at the point of assignment. The following example creates a Workflow from two Pydra Tasks.

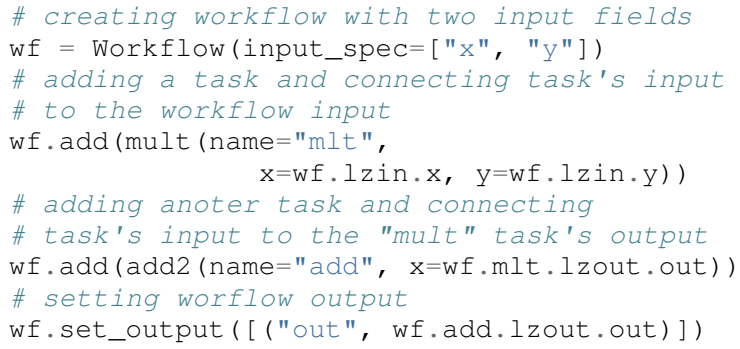

\section{State}

All Tasks, including Workflows, can have an optional attribute representing an instance of the State class. This attribute controls the execution of a Task over different input parameter sets. This class is at the heart of Pydra's powerful Map-Reduce over arbitrary inputs of nested dataflows feature. The State class formalizes how users can specify arbitrary combinations. Its functionality is used to create and track different combinations of input parameters, and optionally allow limited or complete recombinations. In order to specify how the inputs should be split into parameter sets, and optionally combined after the Task execution, the user can set splitter and combiner attributes of the State class. These attributes can be set by calling split and combine methods in the Task class. Here we provide a simple Map-Reduce example:

task_with_state $=$ $\operatorname{add} \overline{2}(x=[1,5])$. split ("x") . combine ("x")

In this example, the State class is responsible for creating a list of two separate inputs, $[\{x: 1\},\{x: 5\}]$, each run of the Task should get one element from the list. The results are grouped back when returning the result from the Task. While this example illustrates mapping and grouping of results over a single parameter, Pydra extends this to arbitrary combinations of input fields and downstream grouping over nested dataflows. Details of how splitters and combiners power Pydra's scalable dataflows are described later.

\section{Submitter}

The Submitter class is responsible for unpacking Workflows and single Tasks with or without State into standalone stateless jobs, runnables, that are then executed by Workers. When the runnable is a Workflow, the Submitter is responsible for checking if the Tasks from the graph are ready to run, i.e. if all the inputs are available, including the inputs that are set to the Lazy Outputs from previous Tasks. Once a Task is ready to run, the Submitter sends it to a Worker. When the runnable has a State, then the Submitter unpacks the State and sends multiple jobs to the Worker for the same Task. In order to avoid memory consumption as a result of scaling of Tasks, each job is sent as a pointer to a pickle file, together with information about its state, so that proper input can be retrieved just before running the Task. Submitter uses AsyncIO to manage all job executions to work in parallel, allowing scaling of execution as Worker resources are made available.

\section{Workers}

Workers in Pydra are responsible for the actual execution of the Tasks and are initialized by the Submitter. Pydra supports three types of execution managers: ConcurrentFutures, Slurm and Dask (experimental). When ConcurrentFuturesWorker is created, ProcessPoolExecutor is used to create a "pool" for adding the runnables. SlurmWorker creates an "sbatch" submission script in order to execute the task, and DaskWorker make use of Dask's Client class and its submit method. All workers use async functions from AsyncIO in order to handle asynchronous processes. All Workers rely on a load_and_run function to execute each job from its pickled state.

\section{Key Features}

In this section, features of Pydra that exemplify its utility for scientific dataflows are presented. Individually, some of these features are present in the numerous workflow packages that exist, but Pydra is the only software that brings them together using a very lighweight codebase. The combination of the following features makes Pydra a powerful tool in scientific computation.

\section{Nested and Hashed Workflows}

Scientific dataflows typically involve significant refinement and extensions as science and instrumentation evolves. Pydra was designed to provide an easy way of creating scientific dataflows that range from simple linear pipelines to complex nested graphs. It enables reproducibility and reduces cost of dataflow maintenance through flexible reuse of already existing functions and Workflows in new applications. The Workflow class inherits 
from TaskBase class and can be treated by users as any other Task, so can itself be added as a node in another Workflow. This provides an easy way of creating nested Workflows of arbitrary depth, and reuse already existing Workflows. This is schematically shown in Fig. 2.

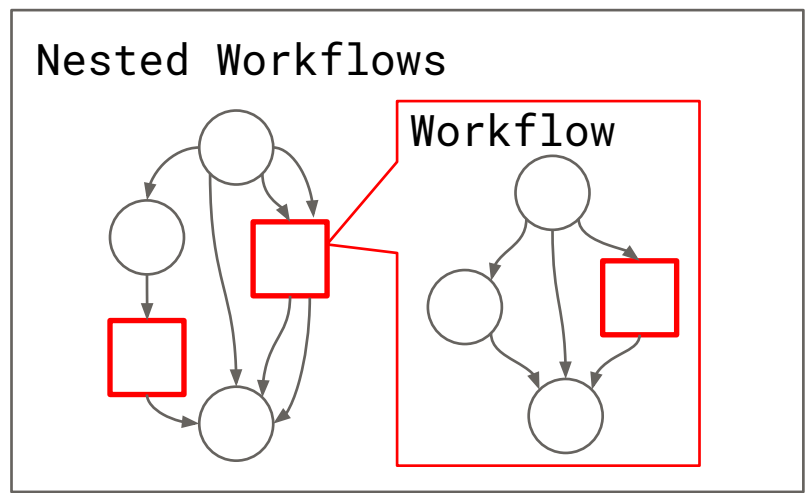

Fig. 2: A nested Pydra Workflow, black circles represent single Tasks, and Workflows are represented by red rectangles.

The Pydra's Submitter supports this nested architecture and can dynamically extend the execution graph. Since a Workflow works like a Task-has inputs, outputs, and is hashable, once executed it does not need to recompute its operations if cached (Pydra's caching is explained later in the section).

\section{State and Nested Loops over Input}

One of the main goals of creating Pydra was to support flexible evaluation of a Task or a Workflow over combinations of input parameters. This is the key feature that distinguishes it from most other dataflow engines. This is similar to the concept of the Map-Reduce [DG04], but extends it to work over arbitrary nested graphs. In complex dataflows, this would typically involve significant overhead for data management and use of multiple nested loops. In Pydra, this is controlled by setting specific State related attributes through Task methods. In order to set input splitting (or mapping), Pydra requires setting up a splitter. This is done using Task's split method. The simplest example would be a Task that has one field $x$ in the input, and therefore there is only one way of splitting its input. Assuming that the user provides a list as a value of $x$, Pydra splits the list, so each copy of the Task will get one element of the list. This can be represented as follow:

$$
S=x: x=\left[x_{1}, x_{2}, \ldots, x_{n}\right] \longmapsto x=x_{1}, x=x_{2}, \ldots, x=x_{n},
$$

where $S$ represents the splitter, and $x$ is the input field.

That is also represented in Fig. 3, where $x=[1,2,3]$ as an example.

Scalar and outer splitters: Whenever a Task has more complicated inputs, i.e. multiple fields, there are two ways of creating the mapping, each one is used for different application. These splitters are called scalar splitter and outer splitter They use a special, but Python-based syntax as described next.

A scalar splitter performs element-wise mapping and requires that the lists of values for two or more fields to have the same length. The scalar splitter uses Python tuples and its operation is therefore represented by a parenthesis, ():

$$
\begin{aligned}
S=(x, y) \quad: \quad x=\left[x_{1}, x_{2}, . ., x_{n}\right], \quad y=\left[y_{1}, y_{2}, . ., y_{n}\right] \\
\mapsto \quad(x, y)=\left(x_{1}, y_{1}\right), \ldots,(x, y)=\left(x_{n}, y_{n}\right),
\end{aligned}
$$

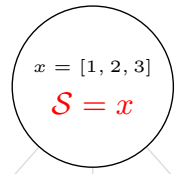

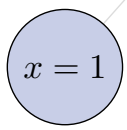

out $=5$

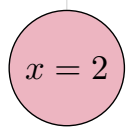

out $=3$

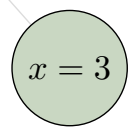

out $=4$
Fig. 3: Diagram representing a Task with one input and a simple splitter. The white node represents an original Task with $x=[1,2,3]$ as an input and $S=x$ as a splitter. The coloured nodes represent stateless copies of the original Task after splitting the input, these are the runnables that are executed by Workers.

where $S$ represents the splitter, $x$ and $y$ are the input fields.

This is also represented as a diagram in Fig. 4
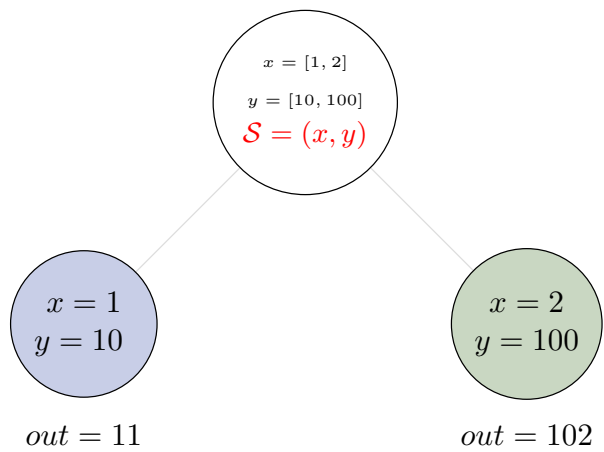

out $=102$

Fig. 4: Diagram representing a Task with two input fields and a scalar splitter. The symbol convention is described in 3.

The second option of mapping the input, when there are multiple fields, is provided by the outer splitter. The outer splitter creates all combination of the input values and does not require the lists to have the same lengths. The outer splitter uses Python's list syntax and is represented by square brackets, [ ] :

$$
\begin{aligned}
S=[x, y] & : \quad x=\left[x_{1}, x_{2}, \ldots, x_{n}\right], y=\left[y_{1}, y_{2}, \ldots, y_{m}\right], \\
& \mapsto \quad(x, y)=\left(x_{1}, y_{1}\right),(x, y)=\left(x_{1}, y_{2}\right) \ldots,(x, y)=\left(x_{n}, y_{m}\right) .
\end{aligned}
$$

The outer splitter for a node with two input fields is schematically represented in Fig. 5

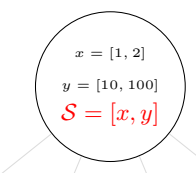

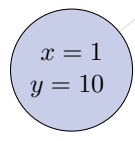

out $=11$

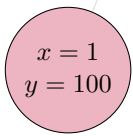

out $=101$

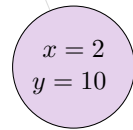

out $=12$

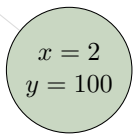

out $=102$
Fig. 5: Diagram representing a Task with two input fields and an outer splitter. The symbol convention is described in 3.

Different types of splitters can be combined over inputs such as [inp1, (inp2, inp3)]. In this example an outer splitter provides 
all combinations of values of inp1 with pairwise combinations of values of inp2 and inp3. This can be extended to arbitrary complexity.

Combiners: In addition to the splitting the input, Pydra supports grouping or combining the output resulting from the splits. Taking as an example the simple Task represented in Fig. 3, in some application it can be useful to group all output values of the individual splits. In order to achieve this for a Task, a user can specify a combiner. This can be set by calling combine method. Note, the combiner only makes sense when a splitter is set first. When combiner $=x$, all values are combined together within one list, and each element of the list represents an output of the Task for the specific value of the input $x$. Splitting and combining for this example can be written as follows:

$$
\begin{aligned}
& S=x \quad: \quad x=\left[x_{1}, x_{2}, \ldots, x_{n}\right] \mapsto x=x_{1}, x=x_{2}, \ldots, x=x_{n} \\
& C=x \quad: \quad \text { out }\left(x_{1}\right), \ldots, \text { out }\left(x_{n}\right) \mapsto \text { out }_{\text {comb }}=\left[\operatorname{out}\left(x_{1}\right), \ldots \text { out }\left(x_{n}\right)\right]
\end{aligned}
$$

where $S$ represents the splitter, $C$ represents the combiner, $x$ is the input field, out $\left(x_{i}\right)$ represents the output of the Task for $x_{i}$, and out comb $_{\text {is }}$ is the final output after applying the combiner.

In the situation where input has multiple fields and an outer splitter is used, there are various ways of combining the output. Taking as an example Task represented in Fig. 5, user might want to combine all the outputs for one specific value of :math: $x \_i$ and all the values of :math:y. In this situation, the combined output would be a two dimensional list, each inner list for each value of :math: $x$. This is written as follows:

$$
\begin{aligned}
C=y \quad: \quad & \text { out }\left(x_{1}, y 1\right), \text { out }\left(x_{1}, y 2\right), \ldots \text { out }\left(x_{n}, y_{m}\right) \\
\longmapsto \quad & {\left[\left[\text { out }\left(x_{1}, y_{1}\right), \ldots, \text { out }\left(x_{1}, y_{m}\right)\right]\right.} \\
& \ldots, \\
& {\left.\left[\operatorname{out}\left(x_{n}, y_{1}\right), \ldots, \text { out }\left(x_{n}, y_{m}\right)\right]\right] . }
\end{aligned}
$$

And is represented in Fig. 6.

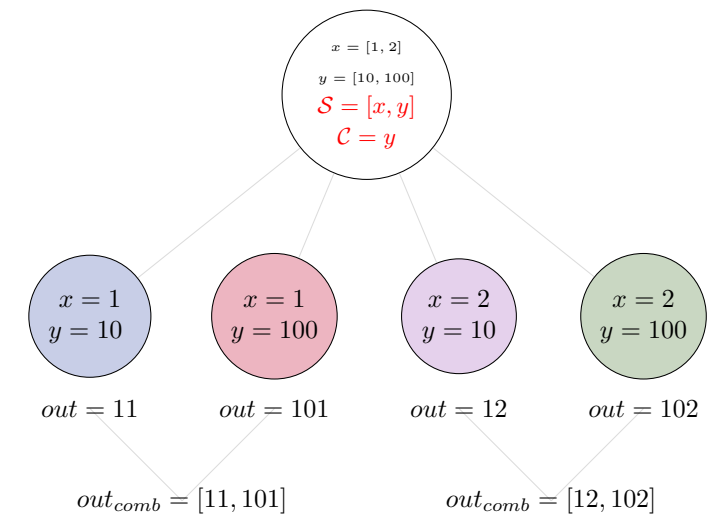

Fig. 6: Diagram representing a Task with two input fields, an outer splitter and a combiner. The white node represents an original Task with $x=[1,2], y=[10,100]$ as an input, $S=[x, y]$ as a splitter, and $C=y$ as a combiner. The coloured nodes represent stateless copies of the original Task after splitting the input, these are the runnables that are executed by Workers. At the end outputs for all values of $y$ are combined together within out comb $_{\text {. }}$

However, for the diagram from 5, the user might want to combine all values of $x$ for specific values of $y$. One may also need to combine all the values together. This can be achieved by providing a list of fields, $[x, y]$ to the combiner. When a full

combiner is set, i.e. all the fields from the splitter are also in the combiner, the output is a one dimensional list:

$C=[x, y]: \operatorname{out}\left(x_{1}, y 1\right), \ldots$ out $\left(x_{n}, y_{m}\right) \longmapsto\left[\operatorname{out}\left(x_{1}, y_{1}\right), \ldots\right.$, out $\left.\left(x_{n}, y_{m}\right)\right]$.

And is represented in Fig. 7.

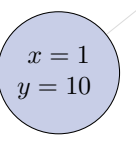

out $=11$$$
\text { (1) }
$$

$$
\text { out }
$$

$$
\text { out }_{\text {comb }}=[11,101,12,102]
$$

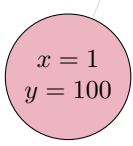

out $=101$

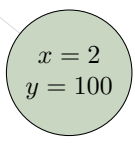

out $=102$
Fig. 7: Diagram representing a Task with two input fields, an outer splitter and a full combiner. The Tasks are run in exactly the same way as previously, but at the end all of the output values are combined together. The symbol convention is described in 6.

These are the basic examples of the Pydra's splitter-combiner concept. It is important to note, that Pydra allows for mixing splitters and combiners on various levels of a dataflow. They can be set on a single Task or a Workflow. They can be passed from one Task to following Tasks within the Workflow. Examples of this more complex operation are presented in the next section.

\section{Checksums and Global Cache}

One of the key feature of Pydra is the support for a Global Cache. This allows multiple people in a laboratory, or even across laboratories to use each other's execution outputs on the same data without having to rerun the same computation. Each Task and Workflow has an attribute called checksum. In order to create the checksum, all of the input fields are collected and hash value is calculated. If File or Directory is used as an input, than the hash value of the content is used. For Workflows, the connections between the Tasks are also included in the final checksum, and hence the checksum of a Workflow changes if its underlying graph changes. The checksum is used to create output directory path during execution and can be reused in future executions of the same exact Task or Workflow. To reuse, a user can specify cache_dir and cache_locations when creating a Task or Workflow. The cache_dir is a read-write path, where you want your outputs to be saved, but cache_location can include a list of paths, which allow re-using existing caches. Before running any Task or Workflow, Pydra checks all the directories that are either in cache_dir or cache_locations, and if the specific checksum is found, then the results are reloaded without running the specific Task. It is important to emphasize that without a cache, every element of a nested Workflow would be re-executed. Using Global Cache can significantly reduce execution time when the same operations on the same data are repeated. This is also true for Tasks with State. If the number of input elements is expanded, the previously cached results can be reused without recomputation. For scientific workflows, where many tasks take significant computational resources, this can drastically speed up reruns. 


\section{Applications and Examples}

In this section, we highlight Pydra through two examples. The first example is an intuitive scientific Python example to demonstrate the power of Pydra's splitter and combiner. The second example extends this demonstration with a more practical machine learning model comparison workflow leveraging scikit-learn.

\section{Example 1: Sine Function Approximation}

This example illustrates the flexibility of the Pydra's splitters and combiners, but the example is not meant to convince scientist to use Pydra to write algorithms like this. The exemplary workflow will calculate the approximated values of Sine function for various values of $x$. The Workflow uses the Taylor polynomial formula for Sine function:

$$
\sum_{n=0}^{n_{\max }} \frac{(-1)^{n}}{(2 n+1) !} x^{2 n+1}=x-\frac{x^{3}}{3 !}+\frac{x^{5}}{5 !}+\ldots
$$

where $n_{\max }$ is a degree of approximation.

Since the idea is to make the execution as embarassingly parallel as possible, each of the term for each value of $x$ should be calculated separately. This is done by function term $(x, n)$. In addition, range_fun(n_max) is used to return a list of integers from 0 to $n \_$max and summing(terms) will sum all the terms for the specific value of $x$ and $n \_m a x$.

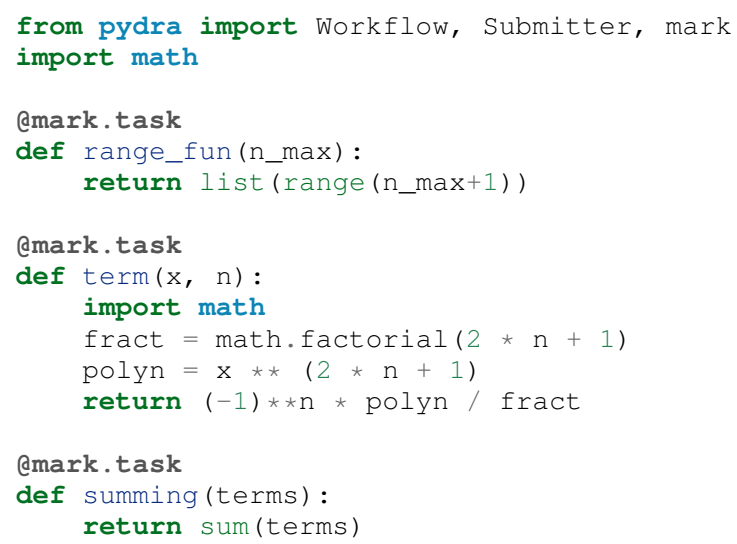

The Workflow takes two inputs - a list of values of $x$ and a list of values of $n \_$max. In order to calculate various degrees of the approximation for each value of $x$, an outer splitter is used [ $\left.x, n \_m a x\right]$. All approximations for a specific values of $x$ is aggregated by using $n \_\max$ as a combiner.

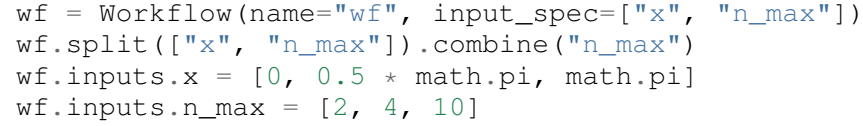

All three Function Tasks are added to the Workflow and connected together using lazy connections. The second task, term, has to be additionally split over $n$ to compute the different pieces of the Taylor approximation and the results of each term calculation are grouped together through the combine method.

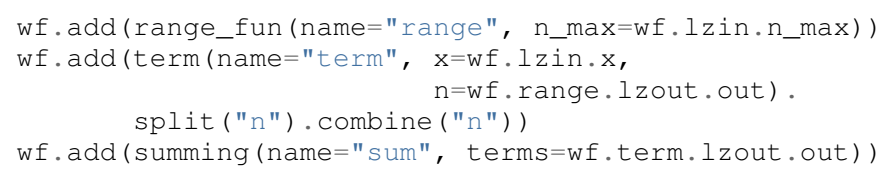

Finally, the Workflow output is set as the approximation using set_output method. Thus the Workflow reflects a parallelizable self contained function. wf.set_output ([ ("sin", wf.sum.lzout.out)]) res $=$ wf (plugin="cf")

When executed using the concurrent futures library, the result is a two dimensional list of Results. For each value of $x$ the Workflow computes a list of three approximations. As an example, for $x=p i / 2$ this returns the following list:

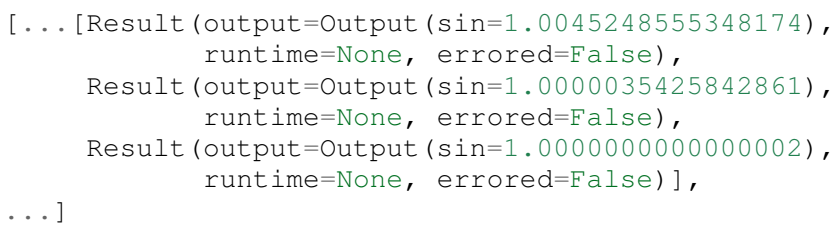

Each Result contains three elements: output reflecting the actual computed output, runtime reflecting the information related to resources used during execution (when a resource audit flag is set), and errored a boolean flag which indicates whether the task errored or not. As expected, the values of the Sine function are getting closer to 1 with increasing degree of the approximation.

The described Workflow is schematically presented in Fig. 8.

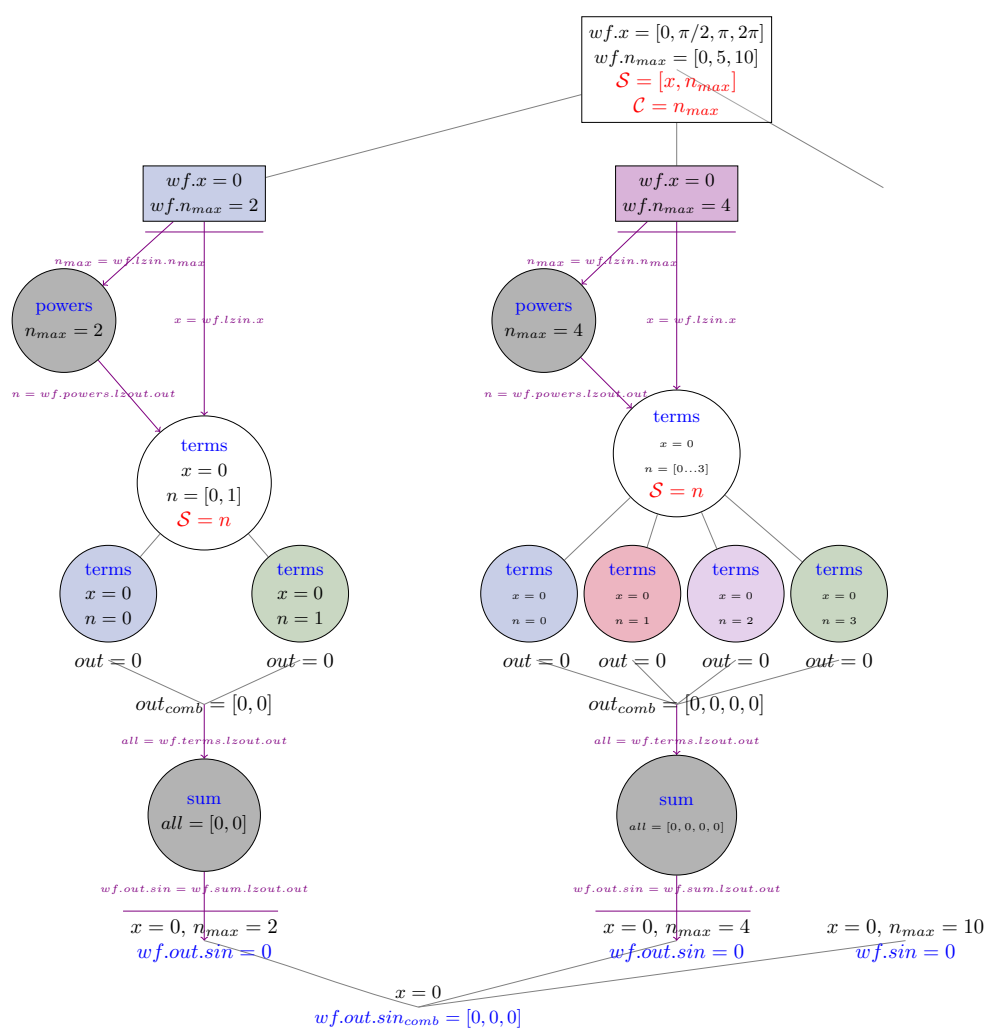

Fig. 8: Diagram representing part of the Workflow for calculating Sine function approximations of various degrees for values of $x$. Circles represent single Tasks and rectangles represent Workflows. The white nodes represent Task or Workflow with a State. The coloured nodes represent stateless copies of the original Task after splitting the input. The gray nodes represent a Task that has no State.

\section{Example2: Machine Learning Model Comparison}

The massive parameter search space of models and their parameters makes machine learning an ideal use case for Pydra. This section illustrates a general-purpose machine learning Pydra's Workflow for model comparison using a boostrapped shuffle-split mechanism for choosing training and test pairs from a given dataset. The example leverages Pydra's powerful splitters and 
combiners to scale across a set of classifiers and metrics. It also uses Pydra's caching to not redo model training and evaluation when new metrics are added, or when number of iterations is increased. The complete model comparison workflow is available as an installable package called pydra-ml [pyd], and includes SHAP-based feature importance evaluation in addition to model comparison.

The Workflow presented here comprises four FunctionTasks. For the sake of clarity, we will not redisplay the task code here. They can be found in the tasks.py file in pydra-ml [pyd]. The first function, read_data, reads csv data as a pandas.DataFrame and allows the user to extract specific columns as the input, $X$, to a learning model, a target column, $y$, and an optional group column. The second function, gen_splits, uses GroupShuffleSplit from sklearn.model_selection to generate a set of train-test splits given n_splits and test_size, with an option to define group and random_state. It returns train_test_splits and split_indices. The main function to train the classifier, train_test_kernel, takes as input a specific train-test split pair, a target variable, a parameter providing information about which classifier to use and whether to generate a null model by permuting the labels. The final function calc_metric returns the value from a scoring function given the actual target and predicted values from the classifier.

These tasks are combined together within a Workflow exploiting splitters and combiners. The Workflow itself has an outer split for clf_info and permute, allowing evaluation of null and non-null models for every classifier. The core model fitting and evaluation function train_test_kernel uses an internal splitter to iterate over all the bootstrapped iterations. Using Pydra, it is possible to split over split_index, that comes from gensplit Task, and run train_test_kernel for each of them without combining. This maintains State which can be used by the calc_metric function to evaluate different scoring methods on the classifier outputs and combine these results back together.

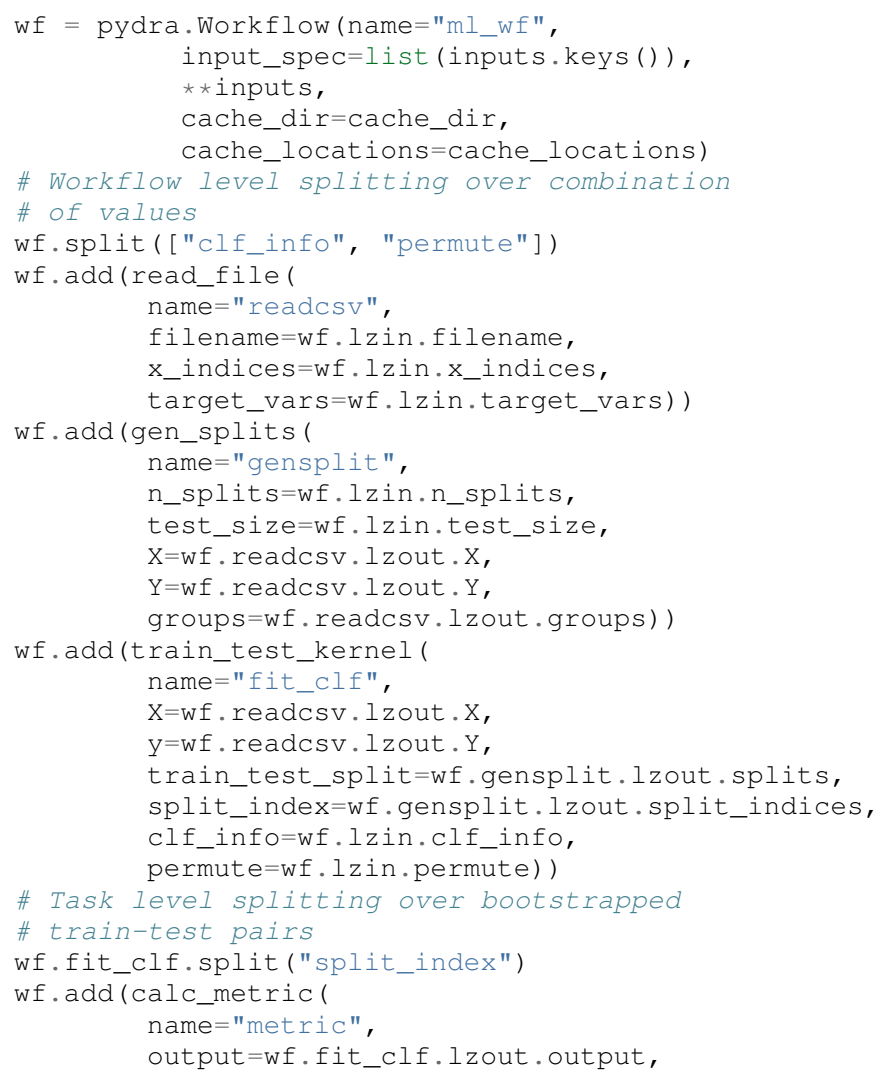

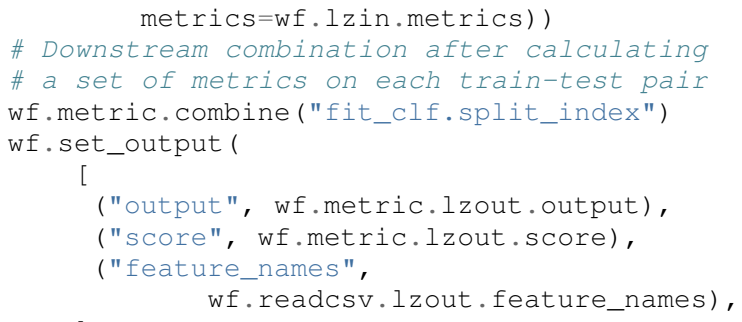

The workflow is executed by providing an input dictionary exemplary input dictionary and the Workflow's submission can look as follow:

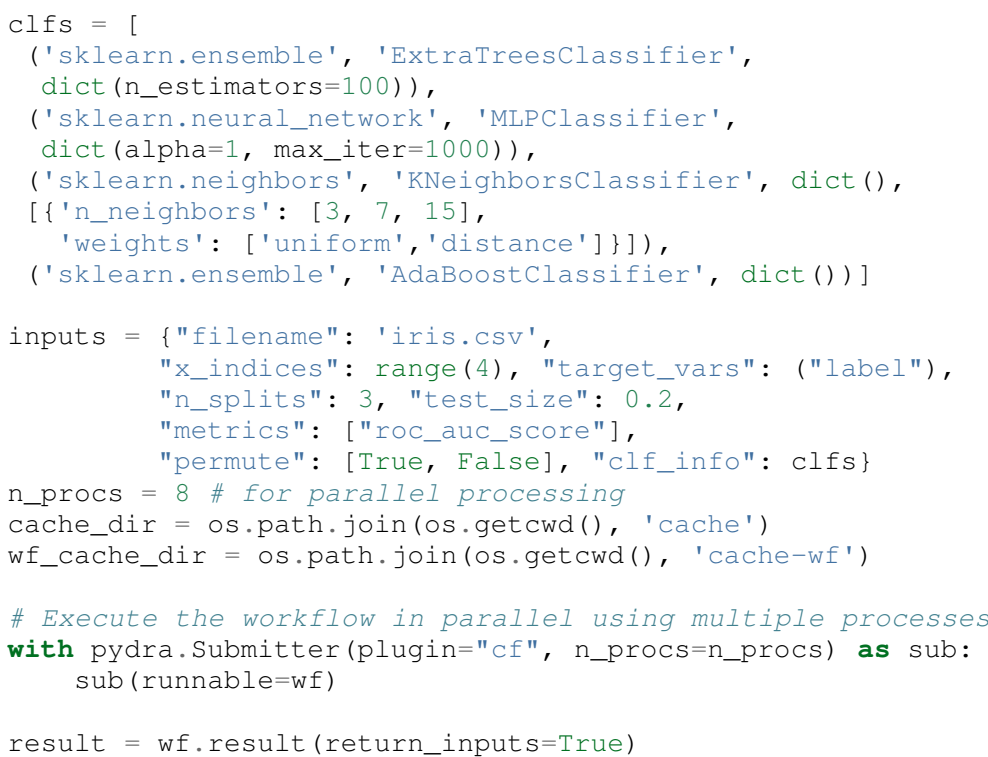

The result from the Workflow is a set of scores for permuted and non-permuted models. This is a list, each element of the list is for one value of clf_info and permute, both fields were set as input fields to the Workflow. All Result objects have an output.score field that is also a list. Each element of the score corresponds to a different value of split_index, that was set both as a splitter and combiner to the fit_cls Task. This gives an option to easily compare various models and sets of parameters.

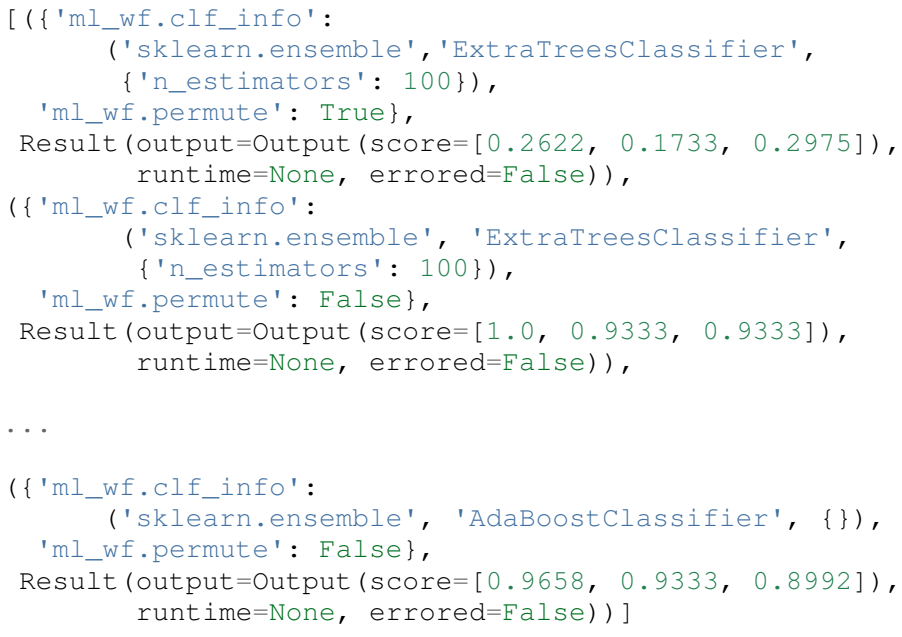

Usually, there is no easy way in scikit-learn to compare models in parallel across a variety of classifiers without using loops. It is possible to do all this natively in scikit-learn and joblib, but would 
require much more code to do the maintenance of the dataflow and aggregation.

\section{Summary and Future Directions}

Pydra is a new lightweight dataflow engine written in Python. The combination of several key features - including flexible option for splitting and combining input fields, and Global Cache makes Pydra a customizable and powerful dataflow engine. The Pydra's developers are mostly from the Neuroimaging community, which provides a plethora of use-cases for complex dataflows, but the package is designed as a general-purpose dataflow engine to support any scientific domain. As the next step, the developer team would like to invite more scientist to use Pydra in order to test the package for diverse applications. In the near future, the developer team is also planning to work on:

- improvement of Worker classes to coordinate resource management

- improved interaction with Dask and other resource managers (e.g., SLURM) in HPC and Cloud environments.

- $\quad$ updates to the Nipype software to use Pydra as its engine

- improve the documentation and tutorials

We welcome scientists and developers to join the project. The project repository is available on GitHub under Nipype organization: https://github.com/nipype/pydra. In addition, there is also a repository that contains Jupyter Notebooks with Pydra tutorial: https://github.com/nipype/pydra-tutorial. The tutorial can be run locally or using the Binder service.

\section{Acknowledgements}

This was supported by NIH grants P41EB019936, R01EB020740. We thank the neuroimaging community for feedback during development, and Anna Jaruga for her feedback on the paper.

\section{REFERENCES}

[ATS09] Brian B Avants, Nick Tustison, and Gang Song. Advanced normalization tools (ants). Insight j, 2(365):1-35, 2009.

[C-P] C-PAC. http://fcp-indi.github.io/.

[Cox96] Robert W. Cox. Afni: Software for analysis and visualization of functional magnetic resonance neuroimages. Computers and Biomedical Research, 29(3):162 - 173, 1996. URL: http://www. sciencedirect.com/science/article/pii/S0010480996900142, doi : https://doi.org/10.1006/cbmr.1996.0014.

[Dev] Nipype Developers.

[DFS99] Anders M. Dale, Bruce Fischl, and Martin I. Sereno. Cortical surface-based analysis: I. segmentation and surface reconstruction. NeuroImage, 9(2):179 - 194, 1999. URL: http://www. sciencedirect.com/science/article/pii/S1053811998903950, doi : https://doi.org/10.1006/nimg.1998.0395.

[DG04] Jeffrey Dean and Sanjay Ghemawat. Mapreduce: Simplified data processing on large clusters. In OSDI'04: Sixth Symposium on Operating System Design and Implementation, pages 137-150, San Francisco, CA, 2004.

[doc] Docker. https://www.docker.com/.

$\left[\mathrm{FAK}^{+}\right.$07] K.J. Friston, J. Ashburner, S.J. Kiebel, T.E. Nichols, and W.D. Penny, editors. Statistical Parametric Mapping: The Analysis of Functional Brain Images. Academic Press, 2007. URL: http: //store.elsevier.com/product.jsp?isbn=9780123725608.

$\left[\mathrm{GBM}^{+}\right.$11] Krzysztof Gorgolewski, Christopher Burns, Cindee Madison, Dav Clark, Yaroslav Halchenko, Michael Waskom, and Satrajit Ghosh. Nipype: A flexible, lightweight and extensible neuroimaging data processing framework in python. Frontiers in Neuroinformatics, 5:13, 2011. URL: https://www.frontiersin.org/article/10.3389/ fninf.2011.00013, doi:10.3389/fninf.2011.00013.
[OEG19] muzpe James D. Kent Mathias Goncalves Elizabeth DuPre Madeleine Snyder Hiroyuki Oya Satrajit S. Ghosh Jessey Wright Joke Durnez Russell A. Poldrack Oscar Esteban, Christopher J. Markiewicz and Krzysztof J. Gorgolewski. fmriprep: a robust preprocessing pipeline for functional mri. Nature Methods, 16:111 - 116, 2019. doi:doi:10.1038/s41592-018$0235-4$.

[pyd] pydra-ml. https://github.com/nipype/pydra-ml.

[sin] Singularity. https://sylabs.io/docs/.

$\left[\mathrm{WJP}^{+}\right.$09] Mark W. Woolrich, Saad Jbabdi, Brian Patenaude, Michael Chappell, Salima Makni, Timothy Behrens, Christian Beckmann, Mark Jenkinson, and Stephen M. Smith. Bayesian analysis of neuroimaging data in fsl. NeuroImage, 45(1, Supplement 1):S173 - S186, 2009. Mathematics in Brain Imaging. URL: http://www.sciencedirect.com/science/article/pii/ S1053811908012044, doi:https://doi.org/10.1016/ j.neuroimage.2008.10.055. 